INTERDISCIPLINARIA ARCHAEOLOGICA NATURAL SCIENCES IN ARCHAEOLOGY

\title{
Generalised Typesets in Experimental Ceramics: Widening Applicability and Maximizing Cross-cultural Assessments
}

\author{
Caroline Jeffra ${ }^{1 *}$ \\ ${ }^{1}$ University of Amsterdam, BG 1, Turfdraagsterpad 9, 1012XT Amsterdam, The Netherlands
}

\section{ARTICLE INFO}

\section{Article history:}

Received: $8^{\text {th }}$ February 2021

Accepted: $19^{\text {th }}$ September 2021

DOI: http://dx.doi.org/10.24916/iansa.2021.2.5

\section{Key words:}

experimental archaeology

experimental methods

ceramic typeset

analogy

potter's wheel

\begin{abstract}
$A B S T R A C T$
Investigations tackling the production techniques used by ancient potters often rely upon experimental archaeology to clarify the relationship between surface morphology, surface topography, and the techniques, methods, and gestures used in the potting process. These experimental archaeology programmes focus on creating collections of experimental vessels to compare against archaeologicallyrecovered vessels, thus allowing production techniques to be identified. Often times, however, the typesets generated are designed to address a specific intersection of qualities; replica vessels adhere to a tight range of shapes, dimensions, paste recipes, and/or forming techniques. As such, the applicability of those typesets remains narrow and context-specific. How, then, can researchers tackle assemblages with diverse vessel types? Or contexts composed of competing potting traditions? Or contexts with significant proportions of vessels from many different origins?

This paper presents a new approach to the way that experimental typesets are designed, developed specifically to address the problem of reliably identifying forming techniques across multiple assemblages. By focusing on accommodating common geometric possibilities of vessel shapes, a generalised typeset can allow individuals to make use of well-documented experimental data. The typeset for the Tracing the Potter's Wheel project was designed for broad applicability, and has been made freely accessible as a reference collection. Through the creation of and comparison against a generalised typeset, heterogeneous assemblages can be better understood and resources can be directed toward answering specific questions. This paper presents the theoretical foundations supporting the concept of a generalised typeset, as well as the practice of using a generalised typeset for analysis.
\end{abstract}

\section{Introduction}

One of the principles which underpins experimental archaeology is the validity of analogy; ensuring that strong analogies are established between experimental variables selected and the archaeological record represented forms the framework against which results are measured. The process of crafting a strong analogy within an experiment design also has the effect of placing an experiment within the continuum ranging between actualistic and scientistic (Outram, 2008). On the one end of this continuum, actualism might aim to explore variables directly impacting the experiment while incorporating many conditions with indirect or unknown impact as well (such as might be the case in

*Corresponding author. E-mail: caroline.jeffra@gmail.com ceramic experiments using locally collected clays while using context-appropriate tools and working in a contextappropriate environment). At the other extreme, scientistic experiments may focus on testing a narrowly restricted set of variables while holding most variables in control (such as ceramic experiments which use commercially available clay to create briquettes with differing proportions of temper in a controlled lab environment).

Fashioning in potting, particularly wheel fashioning, has been investigated with the aid of experimental archaeology to create comparative material to clarify details of archaeological material in a number of studies (Berg, 2009; Courty and Roux, 1995; Gandon et al., 2011; Jeffra, 2013; 2015; Roux and Courty, 1998; Thér, 2015; Thér and Toms, 2016). To date, however, these studies have little addressed their location on the actualistic to scientistic continuum. This 


\author{
Testing a narrowly restricted \\ set of variables while holding \\ most variables in control
}

\section{Exploring variables directly impacting the experiment while incorporating many conditions with indirect or unknown impact as well}

\section{SCIENTISM}

ACTUALISM

\begin{abstract}
Example: nonspecific raw materials as well as standardised tools and firing processes

No single, strong analogy

Moderate analogy strength in comparisons across a broad swathe of material culture
\end{abstract}

Example: reproduction of specific paste preparation, vessel shapes, tools used, and firing processes

Strong analogy for the specific material culture it represents

Weak analogy for other material culture not represented in those choices

Figure 1. The actualism-scientism spectrum.

omission does have interesting implications, however, for a far greater number of studies have focused on identifying wheel fashioning methods and techniques without a basis in tailored experimental archaeology (e.g. Choleva, 2013; Choleva, Jung and Kardamaki, 2020; Gorogianni, Abell and Hilditch, 2016; Knappett, 1999; 2005). These studies (whose high quality is not being debated), lacking new experimental archaeology programmes, rely on the experience of the authors for successful identification of relevant evidence of fashioning methods and techniques. This experience may come from the comparison against existing teaching collections (such as Laboratoire Préhistoire \& Technologie's collections de la technothèque, an online reference collection of over 700 ceramic objects at https://teknotek.pretech.cnrs. $\mathrm{fr} / \mathrm{s} / \mathrm{fr} /$ page/techniques-ceramiques, or Tracing the Potter's Wheel Project's own Information Hub of experimental and archaeological reference material at https://tracingthewheel. eu/database/), or consultation with volumes such as Valentine Roux's excellent recent publication "Ceramics and Society: A Technological Approach" (2019). The foundation of the experiences of non-experimentally derived works, however, is often based on the products of existing experiments. For this reason, it is important that scholars employing experimental approaches explicitly outline the applicability of their products for future analogies, thereby enabling scholars lacking training in application of experimental archaeology to better ascertain applicability of analogies.

In considering the process of comparative typeset creation, experiments which address fashioning can be designed in a way which relates to either end of the actualism-scientism spectrum (Figure 1). Actualistic typesets might reproduce specific paste preparation, vessel shapes, tools used, and firing processes. Each choice within this actualistic experiment serves to strengthen the analogy's applicability to the specific material culture it represents. On the other hand, those choices also serve to weaken the analogy's applicability to other material culture not represented in those choices. Scientistic typesets, meanwhile, might include non-specific raw materials such as commercially-available fine clay, a simple vessel shape such as a cylinder, as well as standardised tools and firing processes. This approach effectively disallows the single, strong analogy reached in the case of actualistic typesets, but vessels within the typeset maintain moderate analogy strength in comparisons across a broad swathe of vessel types.

Given the benefits and the drawbacks of these two extreme examples of experimental typeset, it might be tempting to dismiss scientistic examples as irrelevant. This overlooks a few realities which deserve acknowledgement. Firstly, not all assemblages are homogenous; archaeological sites may have hosted lively exchange of material culture and have diverse types and shapes of ceramics, each formed of distinct paste recipes and fired in different ways. If an experimental programme is to accommodate this heterogeneity while also addressing a range of variables (such as different fashioning methods or techniques), then taking an actualistic approach would necessitate a rather large experimental programme. Managing the scale of an experimental programme is at the heart of the second reality which must be acknowledged. Controlling or exploring many variables within an experiment (or series of experiments) requires significant time and labour costs, which are not accessible to many researchers. Overall, scientistic experiments represent opportunities to accommodate the diversity which characterises some archaeological assemblages as well as opportunities to economise on time and labour investment of research.

Experimental archaeology needs not always be performed at either end of this continuum between actualism and scientism, however, and each approach can be deployed 
meaningfully in order to maintain a balance maximising research resources and reaching strong interpretations of material culture. Furthermore, there are intermediate strategies which can be followed. The author has undertaken one such intermediate strategy in two separate experiments, by developing a generalised typeset.

\section{Background}

Overall, the pattern in the application of experimental archaeology is tied to the actualism-scientism continuum described above. Experiments might address general principles of material, which may or may not be directly linked to a particular assemblage or item. These scientistic experiments allow for mechanical behaviours and material properties in material culture production to be better understood. In some cases, these experiments also allow for material culture production techniques to be interpreted. Conversely, actualistic experiments strongly adhere to conditions which are observed in specific archaeological contexts to address questions which relate directly to those contexts. These actualistic experiments are very well-suited to shedding light on material culture production in those archaeological contexts, but do not necessarily give insights into other behaviours of material culture production. A productive research cycle to follow in archaeological experiments starts with scientistic and progresses toward actualistic work. In this fashion, the conceptual underpinnings of material properties and behaviours can be understood before using actualistic experiments to explore the relationship between material behaviours and human behaviours as expressed in material culture. Working from broad to specific questions allows for conservation of resources as well, by virtue of narrowing potential questions to be addressed in actualistic experiments.

A generalised typeset occupies a space between actualistic and scientistic approaches to archaeological experiments, resulting in vessels which can be readily related to a heterogeneous range of archaeological vessels while still maintaining the potential for strong analogies between typeset and archaeological material. A key concept of this work is that generalised typesets are inherently considered to be one phase within an overall research strategy which is designed to efficiently use resources for maximum applicability. This strategy is based on narrowing the range of unanswered questions by working from broad to specific. Broad questions relate well to scientistic applications of experimental archaeology, and may be thought of as conceptual, i.e. "Can one distinguish between wheel-coiling and wheel-throwing?" Specific questions relate best to actualistic applications of experimental archaeology, and are characterised by their attention to fine details, i.e. "Were stemmed cups found at Telos wheel-coiled or wheelfashioned?" But if one wishes to ask intermediate questions, then one must rely on selective specificity, i.e. "What were

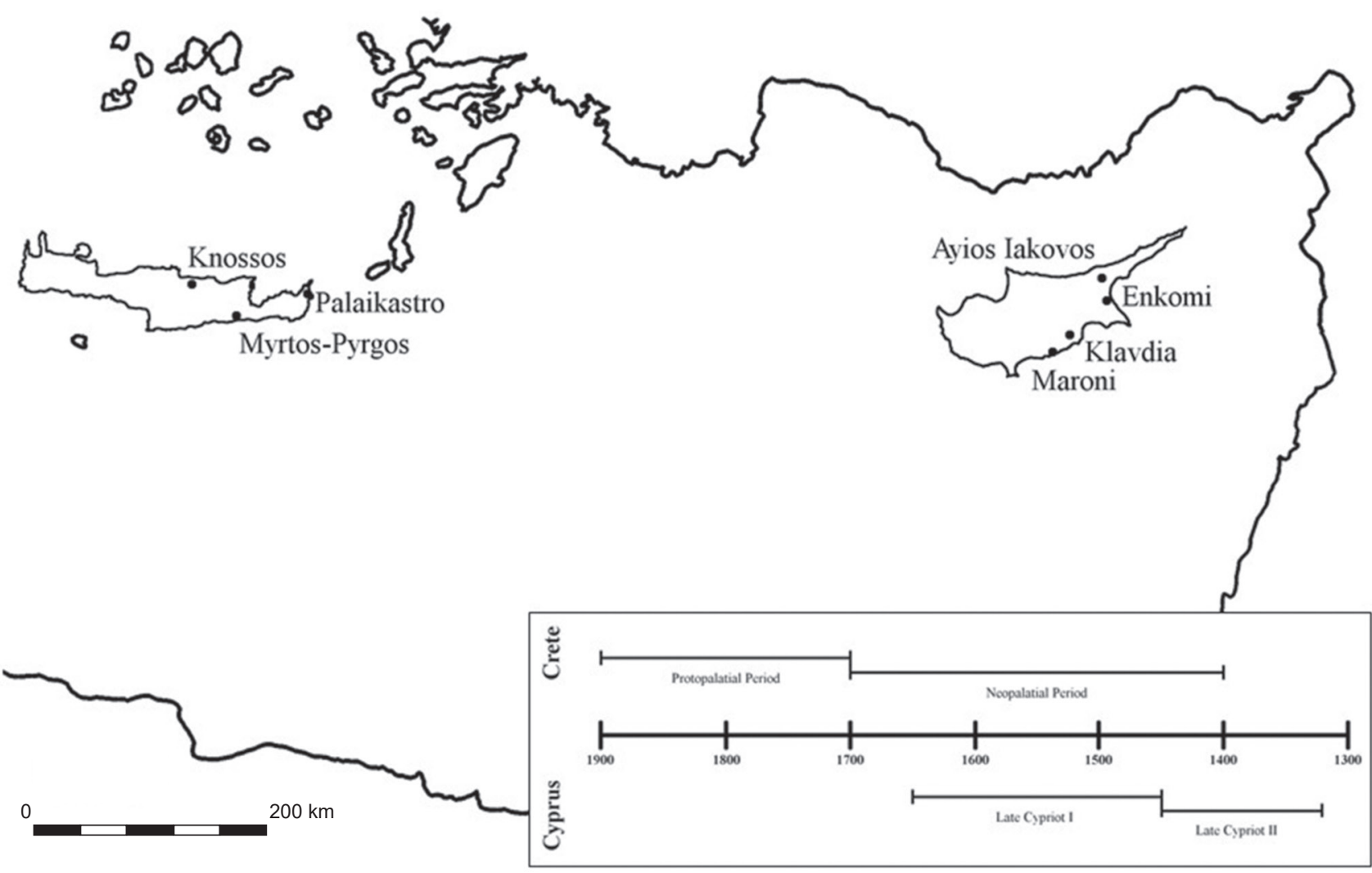

Figure 2. Cretan and Cypriot sites included in pilot study and chronological periods during which time potters were first using the potter's wheel to fashion their pots. 
the fashioning techniques and methods for the assemblage recovered from Telos?"

A major objection to the use of a generalised typeset is that it lacks the specificity of actualistic experimental programmes. This is true; by design a generalised typeset cannot produce the strong analogies which come through exploring many variables to parallel the choices of ancient potters. The strength of a generalised typeset, however, is its suitability for answering most questions about most vessels in an assemblage, which allows for a follow up stage of actualistic experiments to be conducted as needed. There is the added benefit that the subjects of these actualistic experiments are well-justified by virtue of their resistance to identification according to the contents of a generalised typeset.

A generalised typeset, then, occupies an important place between the broad questions of scientistic experiments and the specificity of actualistic experiments. Furthermore, it is the author's position that those producing typesets, particularly generalised typesets, have a responsibility to make them freely accessible. This maximises their benefit to the field by allowing others to progress more readily to either analysing their own assemblages or conducting actualistic experiments of their own.

\section{Method}

Although the concept of using a generalised typeset can be applied to address different experimental variables, the author's focus has been on fashioning methods and techniques. The author's experimental work has largely focused on the time period when potters in Crete and Cyprus were first using the potter's wheel to fashion their pots (Figure 2). As such, the primary concern was to create a typeset which could serve as an adequate analogy for East Mediterranean vessels from the Middle to Late Bronze Age, while also providing comparative material formed using wheel-coiling methods as well as wheel-throwing. The primary variables to be considered and accommodated in establishing the typeset as adequate comparative material were therefore vessel shape and clay texture. These two variable sets had differing implications for the resulting typesets.

\subsection{Accommodating vessel shape}

One of the most readily apparent indices of ceramic heterogeneity is in vessel form. The geometric morphology of vessels is culturally influenced, changing through time and impacted by the ability of potters to replicate specific shapes as needed. Vessel form also should influence the presence and characteristics of forming traces visible on surfaces, differentially revealing those traces during clay deformation operations of vessel shaping. Taking a global view, however, one can argue that although the particulars of vessel form are infinitely customisable, forms are the product of a finite lexicon of geometric shapes. By conceiving of a vessel as a scalable object which is constituted of three zones (lower, middle, upper), those zones can be investigated and the findings from an experimental typeset can be compared against the archaeological material by following a modular approach. Taking this approach, typeset vessels as individuals may poorly reflect the forms represented in an archaeological assemblage, but features of each experimental form can yet be a strong analogy for understanding the fashioning practices represented across a heterogeneous assemblage.

The first pilot of this morphological approach to a generalised typeset was undertaken in experimental work spanning 2007-2011 by the author (Jeffra, 2011). In that work, Cretan and Cypriot excavation reports relating to the earliest periods of wheel use for each context were reviewed. Shapes represented in the typeset were selected based on their morphological dissimilarity from one another as well as each shape's potential for comparison against multiple forms common to the archaeological material.

The most recent application of the morphological approach to creating a generalised typeset was undertaken in the experimental work for the Tracing the Potter's Wheel project (2017-2021) by the author. Unlike the pilot experimental work detailed above, the archaeological sites against which the typeset would be compared were not determined prior to commencing the experimental work, though the project's focus area included Greece, particularly the sites within the Aegean Sea. It was therefore not possible to narrow the range of relevant forms by reviewing excavation reports as was the case in the pilot study. Instead, the process of modular analysis was selected as a strategy to allow experimental work to proceed. To create the conditions for modular analysis of vessels, a broad survey of characteristic as well as rare Bronze Age Aegean ceramic forms was undertaken.

The lower zone of a vessel most significantly includes the inflection point where vessel wall meets base, and for the purposes of this approach incorporates the lowest onefifth to one-third of the vessel wall height. This wall-to-base transition can be characterised by the angle at which wall meets base in the vessel interior, with angles ranging from acute to obtuse. The middle zone of the vessel presents several opportunities for variation, and for the purposes of this approach ranges from the middle one-third to three-fifths of the overall wall height. The morphology of the wall in this middle zone can be characterised based on the presence and nature of the curvature along the vertical axis. Like the middle zone, the upper zone of the vessel can also include different types of curvature along the vertical axis. The region considered the upper of the vessel, for the purposes of this application, included the uppermost one-fifth to onethird of the overall vessel height.

\subsection{Accommodating different clays}

As the research conducted when the generalised typeset was designed centred on East Mediterranean ceramics from the Middle to Late Bronze Age, it was useful to understand the extent of potential heterogeneity of clay recipes in advance. The geology of the East Mediterranean is notoriously 
diverse, and the clays available to and used by potters were correspondingly diverse, from clay body to inclusions to preparation procedures. One aspect the different types of ceramics have in common, however, is the presence of both 'fine' and 'coarse' objects in the archaeological assemblages. Fine ceramics produced at a site in east Crete might have a different texture and colour from those produced at a site in southwest Cyprus, but those objects would hold in common that the clay bodies from which they were formed was well levigated and lacked non-plastic inclusions. Similarly, the raw materials which contributed to the inclusions in coarse ceramics might differ from one production practice to another, but the fact that non-plastic inclusions were well represented in the ceramics was a common feature of those objects. As such, creating a generalised typeset required acknowledging that untempered and tempered clay could produce distinct or differing surface traces indicative of fashioning methods or techniques, and as a result those two clay types should each be represented in the typeset.

The pilot experimental study made use exclusively of fine, untempered clay to form the typeset, while the later study included fine, untempered, as well as fine clay, tempered, with $2.0 \mathrm{~mm}$ grog. While the untempered clay could be taken as representative of similarly untempered ceramics, the grog tempered clay allowed for the role of non-plastic inclusions to be better illustrated within the typeset. This in turn increases the strength of the analogies drawn between experimental and archaeological materials.

\subsection{Other variables}

Although vessel morphology and clay recipe were the two attributes which were most well-considered in attempting to refine the strength of analogy regardless of archaeological context, several other variables are worthy of mention. First of these is the choice of tools. While a generalised typeset approach could accommodate exploration of this variable through use of one or more wheel device types, for example, in the creation of the typesets described herein, an anticlockwise rotating electric wheel was used. This is also the case for smaller hand-held tools such as ribs for scraping and finishing, cloths or sponges for smoothing and adding or removing water from the clay surface, as well as strings or wires used to cut clay (notably from the wheel head, leaving characteristic traces).

Beyond the question of tools as variables, some raw material properties can be explored in a generalised typeset to the benefit of a number of contexts. One such property is the relative humidity and workability of the clay being used. Even viewing potters at work in small and medium-scale modern workshops reveals that clay consistency for wheelfashioning techniques and methods can vary considerably (see, for example, the diversity on show in the video playlist curated to accompany the conference Archaeological Approaches to the Study of the Potter's Wheel: https:// youtube.com/playlist?list=PLqWUT1pOFU1NOY OTD2xFVmPyUG8tvIZF). While this variation is clear, the relationship between clay consistency and traces observable on the finished vessels is less so. Addressing this variation within a generalised typeset could include testing one clay recipe under differing levels of hydration, for example.

\subsection{Creating the generalised typeset}

The generalised typeset concept was, as mentioned above, developed through the completion of a pilot study followed by a later, and more comprehensive, study. The primary aim driving the creation of each typeset was to create a body of material against which archaeological material could be compared in order to establish the fashioning method or technique used to produce those archaeological vessels. As such, this category of variable was the focal point
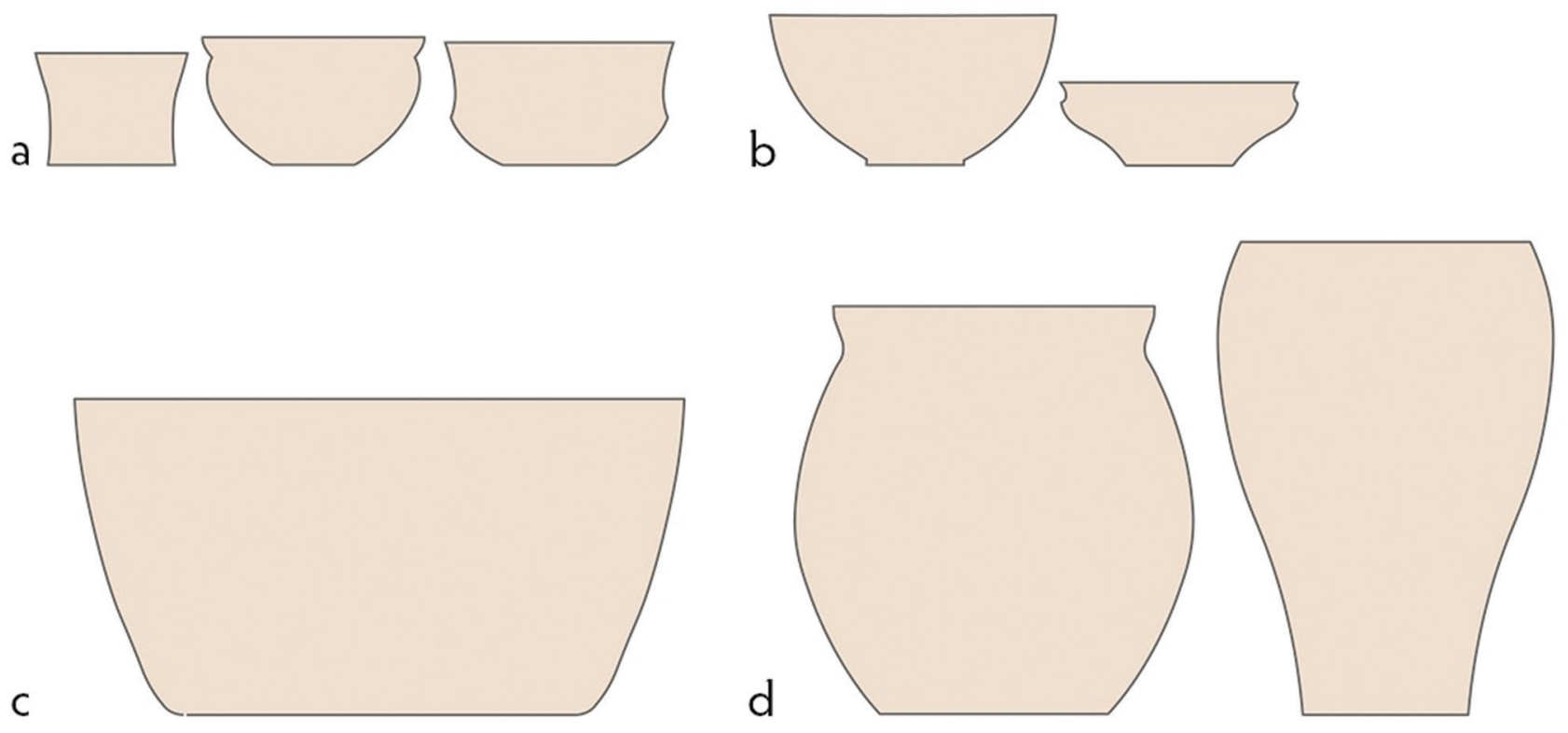

Figure 3. The pilot study found that the assemblages could be represented by a total of eight shapes. 
during each of the experiments. While the pilot experiment focused on differentiating between three of the combination wheel-fashioning methods described by Roux and Courty (1998), the latter experiment involved fashioning vessels with wheel-throwing as well as a specific variation of one wheel coiling method (Roux and Courty's Method 3).

Focusing as it did on two specific island contexts with distinctive ceramic traditions, the pilot study found that the assemblages could be represented by a total of eight shapes (Figure 3). In this first study, it was necessary to economise production to suit the assemblages of the two distinct range of shapes found in Crete and Cyprus during the studied time periods. As such, common features were sought between the two. For smaller vessels, there was a focus on including small, short forms with roughly equal height and rim diameter measurements (Figure 3a) and medium, short forms with wide rim diameters relative to overall height (Figure $3 b$ ). The larger vessels included large, wide forms with wide rim diameters relative to the overall height (Figure 3c) as well as variations on large, tall forms with smaller rim diameter than vessel height dimensions (Figure 3d). Within this pilot, there were no specific expectations about the nature of production traces which might be seen, simply that vessel form and scale may influence the nature of those traces at the macroscopic scale.

For the pilot generalised typeset, wheel-coiling fashioning method and vessel shape were explored, which allowed for a better understanding of how those two variables relate to one another. Each of the eight vessel shapes included was constructed using each of the three wheel-coiling fashioning methods, producing a total of 24 possible combinations. Each of these combinations was produced five times in order to accommodate the probability that relevant forming traces might not appear on every example, resulting in a typeset comprised of 120 individual vessels.

For the fuller experimental generalised typeset, the variables of fashioning technique, clay texture, and vessel shape were explored. In contrast to the pilot typeset, this fuller study was designed more explicitly for wider applicability in terms of vessel shapes. From the survey of Bronze Age Aegean ceramic forms, it was determined that vessel lowers, middles, and uppers most often fell into a handful of geometric groups. In this application, three different variations for vessel lowers were explored: acute, right, and obtuse angles. The traits identified as meaningful for the middle zone of the vessel in this application included convex, straight, and concave. The upper zone possibilities considered for this application included incurving, straight, or everted. These features were combined in ways which found stronger analogies in the east Mediterranean and specifically Aegean Bronze Age contexts. For example, the small vessel with straight sides holds strong morphological similarity to tall conical cups. Overall, five different vessel shapes were included in the creation of the typeset for the TPW project (Figure 4). Each of the five vessel shapes was constructed using either wheel-coiling Method 3 or wheel-throwing, and was formed of either of the two clay types. Each of these 20 combinations of form, fashioning, and clay was created five times, producing a typeset of 100 individual vessels.

\subsection{Using the generalised typeset}

The generalised typeset is best suited for comparison against heterogeneous assemblages. The typeset represents one step in the overall comparative research cycle which finds ever-more-precise analogy to determine potting practices to reveal the details of the chaîne opératoire. In order to use the generalised typeset for establishing fashioning technique, the modular consideration of vessel zones must be revisited. The first priority for establishing strong analogies between experimental typeset and archaeological material is to compare experimental vessel forms which have similar morphology to archaeological examples. In the absence of these direct comparisons, archaeological vessels can be understood by contrasting the lower, middle, and upper zones of their profiles against their experimental counterparts.

This more recent generalised typeset is currently available to access in the Tracing the Potter's Wheel information hub, presented in high quality photographs, downloadable 3D models, video footage of the potting process, and detailed metadata on observed macroscopic traces. This open access data has been made publicly available in order to allow researchers to readily benefit from the application
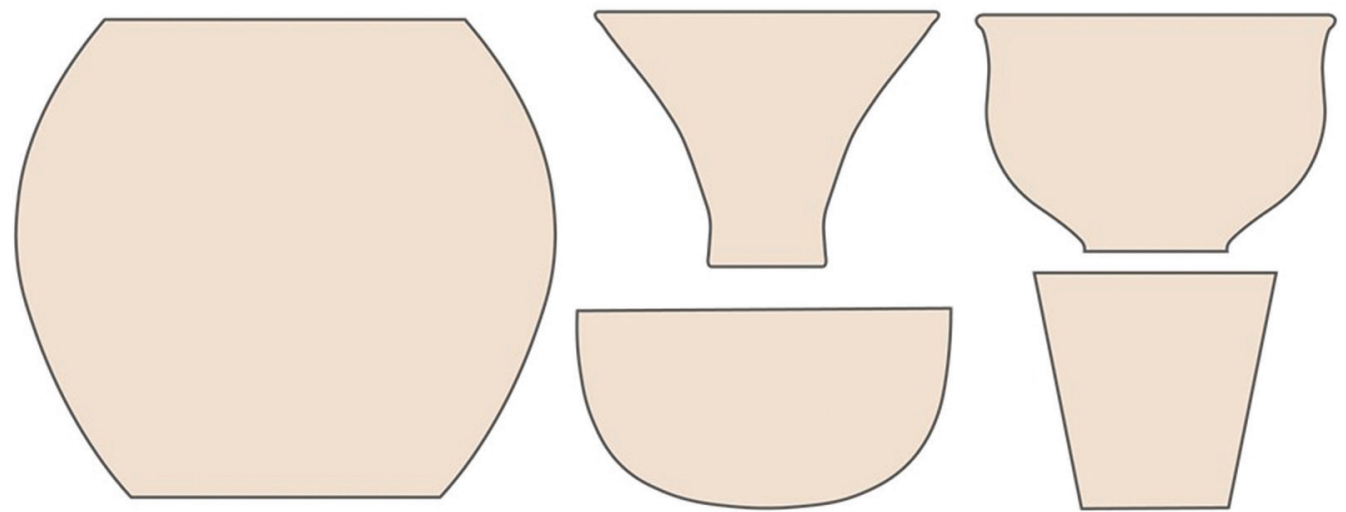

Figure 4. Five different vessel shapes were included in the creation of the typeset for the TPW project. 
of analogies, regardless of their ability to conduct their own experimental research. For those who are able to conduct experimental research, the generalised typeset can represent one burden of labour which they need not bear, freeing up resources to conduct the more precise, actualistic experiments on ceramics whose fashioning remains as open-ended questions.

\section{Conclusion}

By employing a generalised typeset as an analogy to better understand aspects of a heterogeneous assemblage, it is possible to extend the relevance of research endeavours and contribute to a wider world of collaborative scholarship, while also economising on labour investment. A generalised typeset is but one component within the broader picture of investigations into the chaine opératoire of pottery production. Elsewhere, the author has advocated for experiments to be explicitly situated according to their applicability to aspects of and relevance to the chaine opératoire (Jeffra, 2014), a point which equally applies to the present work's focus. What is a generalised typeset good for? Plainly speaking, its main assets are its potential for maximising the effort invested in completing experimental work. If experimental archaeologists are given the opportunity to ask new questions and only duplicate work when the findings themselves are being verified, then subsequent experiments will make incremental progress on elucidating further aspects of the chaîne opératoire. In seeking analogies on which to build an understanding of past material culture production, previously conducted experiments rarely offer a 'perfect fit' for different contexts. The response to accommodating heterogeneous assemblages described herein offers an opportunity for - if not quite a 'perfect fit' - then at least a starting point from which a 'perfect fit' analogy can be established.

\section{References}

BERG, I., 2009. X-Radiography of Knossian Bronze Age Vessels: Assessing our Knowledge of Primary Formation Techniques. Annual of the British School at Athens, 104, 137-174.

CHOLEVA, M., 2013. The First Wheelmade Pottery at Lerna: WheelThrown or Wheel-Fashioned? Hesperia: The Journal of the American School of Classical Studies at Athens, 81, 343-381.

CHOLEVA, M., JUNG, R., and KARDAMAKI, E., 2020. Working on the potter's wheel: technological insights into the Mycenaean pottery production. Egypt and the Levant, 30, 219-282. DOI: 10.1553/ AEundL30s219

COURTY, M.A., and ROUX, V., 1995. Identification of Wheel Throwing on the Basis of Ceramic Surface Features and Microfabrics. Journal of Archaeological Science, 22, 17-50.

GANDON, E., CASANOVA, R., SAINTON, P., COYLE, T., ROUX, V., BRIL, B., and BOOTSMA, R.J., 2011. A proxy of potters' throwing skill: ceramic vessels considered in terms of mechanical stress. Journal of Archaeological Science, 38, 1080-1089.

GOROGIANNI, E., ABELL, N., and HILDITCH, J., 2016. Reconsidering Technological Transmission: The Introduction of the Potter's Wheel at Ayia Irini, Kea, Greece. American Journal of Archaeology, 120(2), 195220. DOI: 10.3764/aja.120.2.0195

JEFFRA, C., 2011. The Archaeological Study of Innovation: An Experimental Approach to the Pottery Wheel in Bronze Age Crete and Cyprus. Exeter: University of Exeter.

JEFFRA, C., 2013. A Re-Examination of Early Wheel Potting in Crete. The Annual of the British School at Athens, 108, 31-49.

JEFFRA, C., 2014. Experimental approaches to archaeological ceramics: unifying disparate methodologies with the chaîne opératoire. Archaeological and Anthropological Sciences, 2014. DOI: 10.1007/ s12520-014-0177-4.

JEFFRA, C., 2015. Experimenting wheel-coiling methods. The Arkeotek Journal, 2015(2). Online: http://www.thearkeotekjournal.org/tdm/ Arkeotek/fr/articles originaux/2Jeffra.xml

KNAPPETT, C., 1999. Tradition and Innovation in Pottery Forming Technology: Wheel-Throwing at Middle Minoan Knossos. Annual of the British School at Athens, 94, 101-129.

KNAPPETT, C., 2005. Artworks and Artefacts: The Pottery from Quartier $\mathrm{Mu}$, Mallia. In: I. Bradfer-Burdet, B. Detournay, R. Laffineur, eds. Kris Technitis / L'Artisan Crétois: Recueil d'Articles En l'Honneur de JeanClaude Poursat, Publié à l'Occasion Des 40 Ans de La Découverte Du Quartier $M u$. Liège: Université de Liège, Histoire de l'art et archéologie de la Grèce antique, pp. 109-118.

OUTRAM, A.K., 2011. Introduction to experimental archaeology. World Archaeology, 40, 1-6. DOI: 10.1080/00438240801889456

ROUX, V., 2019. Ceramics and Society: A Technological Approach to Archaeological Assemblages. Cham, Switzerland: Springer International Publishing.

ROUX, V., and COURTY, M.A., 1998. Identification of Wheel-Fashioning Methods: Technological Analysis of $4^{\text {th }}-3^{\text {rd }}$ Millennium BC Oriental Ceramics. Journal of Archaeological Science, 25, 747-763.

THÉR, R., 2015. Identification of pottery-forming techniques using quantitative analysis of the orientation of inclusions and voids in thin sections. Archaeometry, 58(2), 222-238.

THÉR, R., and TOMS, P., 2016. Quantification of the orientation and alignment of aplastic components of a ceramic body as a method for distinguishing among various means of using a rotational device in pottery forming. Journal of Archaeological Science. Reports, 9, 33-43. DOI: 10.1016/j.jasrep.2016.06.048 
